\title{
Non-linear evolution of uni-directional focussed wave-groups on a deep water: A comparison of models
}

\author{
Thomas A. A. Adcock ${ }^{a}$, Paul H. Taylor ${ }^{a}$ \\ ${ }^{a}$ Department of Engineering Science, University of Oxford
}

\begin{abstract}
Up until the point at which ocean waves break, their dynamics are generally assumed to be accurately modelled by potential flow theory. For practical and computational reasons it is often useful to approximate the full potential flow solution with bandwidth and amplitude limited equations. A approximation used for waves on deep water is the Broad-banded Modified Non-linear Schrödinger equation (also known as the modified Dysthe equation). In this paper we compare this approximate model with potential flow simulations of focussing uni-directional wave-groups. We find that for moderate non-linearity the approximate model predicts very similar changes to the potential flow model. However, one of the dominant non-linear changes to the wave-group is a localised increase in the bandwidth and contraction in physical length, and beyond a certain point the approximate model fails to accurately reproduce this causing other elements, such as the maximum wave amplitude, to be poorly modelled. This modelling inaccuracy occurs in cases where, based on the initial conditions of the simulation, the approximate model would be expected to be accurate.
\end{abstract}

Keywords: Ocean waves; non-linear Schrödinger equation; Dysthe equation; rogue wave; freak wave.

\section{Introduction}

The evolution of ocean waves is a weakly non-linear phenomenon until close to the point at which waves break. A variety of models have been proposed to describe this evolution. In this paper we assume that the evolution of waves (up until breaking) can be accurately described by numerical solutions to the potential flow equations using standard boundary conditions at the free surface which we describe as the 'fully non-linear' model. We compare fully non-linear results to numerical solutions an approximate model which simulates the evolution of the complex wave envelope using higher order extensions of the non-linear Schrödinger (NLS) equation.

Clamond et al. [1] carried out a comparison of the different models examined in this paper but their work focussed on long time-scales rather than the detailed structure of waves locally tall and spatially concentrated wave groups as studied here. Comparisons were also made between the NLS and potential flow simulations by Henderson et al. [2]. Comparisons of the higher order non-linear Schrödinger equation to experiments were made by Shemer \& Dorfman [3] and Lo \& Mei [4]. A similar study to ours - comparing the 

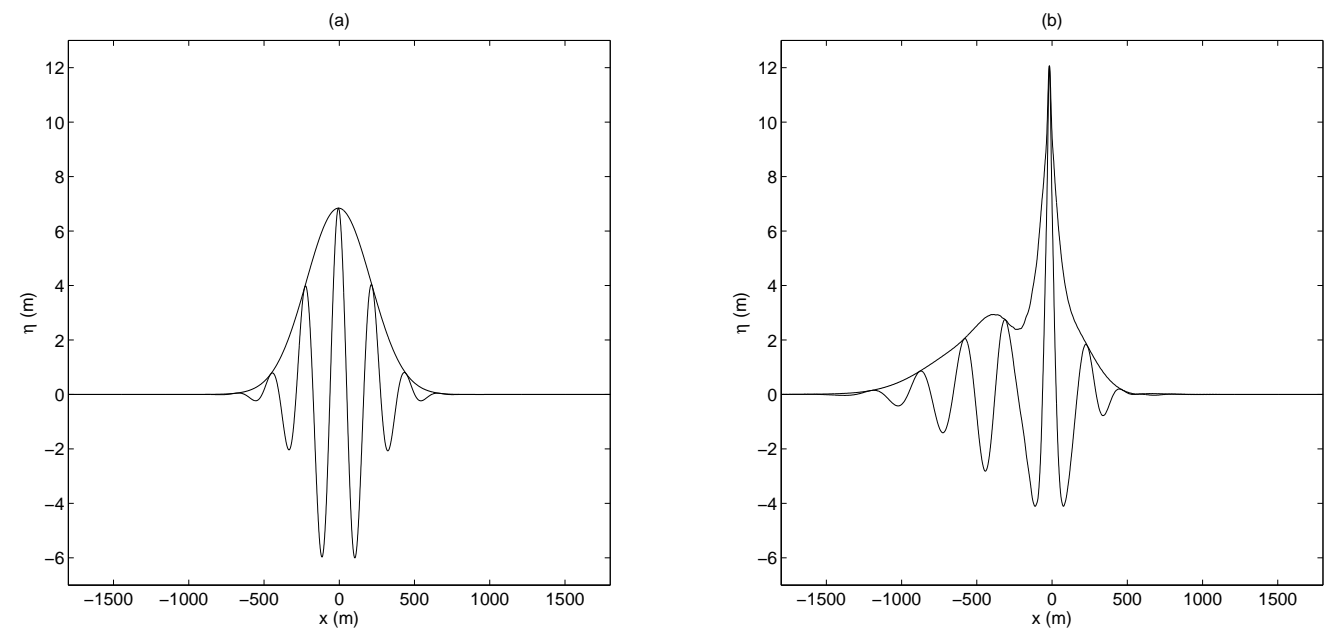

Figure 1: Example of the non-linear changes to a wave-group using fully non-linear model. Both free surface and envelope shown with bound harmonics removed. (a) - linear; (b) - fully non-linear.

approximate envelope model against an exact numerical model - was carried out by Shemer et al. [5] comparing the Zakharov equations, broadbanded NLS, and experiments. This later paper found good agreement for narrow bandwidths between the Zakharov equations and the broadbanded NLS and explored the bandwidth limitations of this. Our study pursues this theme, comparing results from the broadbanded NLS equation against those from a high order spectral scheme for the potential flow equations.

To compare the two models we run simulations of focussed wave-groups. Studying the non-linear changes to isolated wave-groups has been used to investigate non-linear wave evolution by numerous authors using physical experiments [6, 7], numerical models [8, 9, 10], and analytically $[11,12]$. In uni-directional waves non-linearity leads to significant changes to the shape of the wave-group - relative to linear evolution non-linear groups become taller and narrower with the largest wave in the group moving towards the front of the wave-group. An example of this is shown in Figure 1. The formation of this very sharp peak in both the wave envelope and also an individual crest may be compared to the prediction by Lighthill [13] for the changes in both local amplitude and wavenumber as a modulated Stokes wave train evolves. Lighthill predicted the formation of a cusp in the wave envelope; his analysis being based on Whitham's theory [14] for nonlinear-systems where frequency dispersion and amplitude dispersion would be expected to be in competition.

This paper only examines the evolution of uni-directional waves. In the real ocean deepwater waves are not uni-directional but are directionally spread. This fundamentally changes the non-linear processes by which large waves form [15] and means that great caution must be used when applying the results of uni-directional modelling to real ocean waves. In particular, the extra amplitude observed in uni-directional waves greatly reduced in waves with a realistic directional spreading. Nevertheless, uni-directional waves are an important limiting case for the evolution of real ocean waves and are often used in numerical and 
physical experiments for practical reasons. An extension of the results here to directionally spread wave-groups is presented in Adcock \& Taylor [16].

\section{Method}

In this paper we compare fully non-linear potential flow simulations with results solving the broadbanded modified non-linear Schrödinger equation. Our test cases study the focussing of isolated wave-groups on deep water.

We take as our initial conditions a wave-group which, under linear evolution would form a 'NewWave' wave-group 80 periods later. The NewWave is the expected shape of a large wave in a random sea state (see Lindgren [17] and Boccotti [18]) and, at 'focus' $(t=0)$ is given by

$$
\eta(x)=a \frac{\sum_{n} S\left(k_{n}\right) \cos \left(k_{n} x\right)}{\sum_{n} S\left(k_{n}\right)},
$$

where $S\left(k_{n}\right)$ is the discretised wavenumber spectrum of the underlying sea-state and $a$ is the amplitude of the wave-group which can specified arbitrarily. The shape of the linear NewWave group is shown in Figure 1a. This approach has been widely used to study nonlinearity of large waves and Adcock et al. [19] found that analysis of isolated wave-groups closely matched the non-linear changes observed to large waves in random wave-fields.

For these simulations we use a Gaussian wavenumber spectrum which has the shape

$$
S(k)=\lambda \exp \left(\frac{-\left(k-k_{p}\right)^{2}}{2 s_{x}^{2}}\right)
$$

where $k_{p}$ is the peak wavenumber and $s_{x}$ is the bandwidth. The parameter $\lambda$ scales the spectrum although as we only use the shape of the spectrum in this paper this factor does not reappear. In this study we use $k_{p}=0.0279 \mathrm{~m}^{-1}$ (a wavelength of $225 \mathrm{~m}$ ) and $s_{x}=0.0046 \mathrm{~m}^{-1}$. The spectral bandwidth is chosen by fitting the spectral peak of a JONSWAP spectrum with $\gamma=3.3$ with the lower amplitude high frequency tail removed. As such this bandwidth is of the same order of magnitude as would be found in a winter storm in the North Sea. To classify the runs we use the non-dimensionalised amplitude $\left(a k_{p}\right)$ that the wave-group would reach under linear evolution. The most non-linear case we consider would have focussed with $a k_{p}=0.18$. Allowing for the truncation of the high spectral tail, a wave of this steepness might be appropriate for a 1 in 1000 wave in a limiting steepness sea-state. Figure 2 in Socquet-Juglard et al. [20] demonstrates that a limiting steepness of $H_{S} k_{p}=0.36$ occurs in the northern North Sea. Assuming a Rayleigh-type linear crest distribution in a severe sea-state and the truncation of the upper spectral tail, a maximum NewWave individual steepness of $a k_{p}=0.18$ is plausible.

The numerical solution to the potential flow equation uses the high-order pseudo-spectral numerical scheme developed by Bateman et al. [21]. The results used in this paper are taken from Gibbs [22] where they are analysed in depth. As with any numerical solution the results will not be exact, however great care was taken with the simulations and for the purposes of this paper we assume that the results of these simulations can be taken as a benchmark 
to compare against approximate models. The results in this paper used a spatial resolution of seven times the peak wavenumber, a time step of 0.02 seconds with an Adams-Bashford scheme with the equivalent peak wave period $T_{p}=12 \mathrm{~s}$, this gives 97 time-steps per period. The length of the ocean in the assumed periodic computational domain is $8.0 \mathrm{~km}$ with 1024 spatial points. A 7th order expansion of the Dirichlet-Neumann G-operator is used [21]. Some filtering was applied to the highest wavenumber using the 5-point smoothing function of Dommermuth \& Yue [23].

Higher order extensions to the non-linear Schrödinger equation were derived in a series of papers by Dysthe \& Trulsen [24, 25]. The equations model the complex wave envelope, $A$, of the freely propagating waves. The free surface may be recreated from the linear components and narrow bandwidth approximations to the 2nd and 3rd order bound components

$$
\eta=\Re\left(\eta_{\text {linear }}+\eta_{2-}+\eta_{2+}+\eta_{3}\right)
$$

where

$$
\begin{aligned}
\eta_{2-} & =\frac{1}{2 \omega} \frac{\partial \phi}{\partial x}-\frac{1}{16 k} \frac{\partial^{2}|A|^{2}}{\partial x^{2}}, \\
\eta_{\text {linear }} & =A \exp (i(k x-\omega t)), \\
\eta_{2+} & =\left[\frac{k A^{2}}{2}-\frac{i A}{2} \frac{\partial A}{\partial x}\right] \exp (2 i(k x-\omega t)), \\
\eta_{3} & =\frac{3}{8} k^{2} A^{3} \exp (3 i(k x-\omega t)) .
\end{aligned}
$$

The evolution equation is given by

$$
\begin{aligned}
\frac{\partial A}{\partial t}+\frac{\omega}{2 k} \frac{\partial A}{\partial x}+i \frac{\omega}{8 k^{2}} \frac{\partial^{2} A}{\partial x^{2}} & -\frac{\omega}{16 k^{3}} \frac{\partial^{3} A}{\partial x^{3}}-i \frac{5 \omega}{128 k^{4}} \frac{\partial^{4} A}{\partial x^{4}}+\frac{7 \omega}{256 k^{5}} \frac{\partial^{5} A}{\partial x^{5}}= \\
& -\frac{i \omega k^{2}}{2} A|A|^{2}-\frac{3}{2} \omega k|A|^{2} \frac{\partial A}{\partial x}-\frac{1}{4} \omega k A^{2} \frac{\partial A^{*}}{\partial x}-\left.i k A \frac{\partial \phi}{\partial x}\right|_{z=0} .
\end{aligned}
$$

where $k$ is the characteristic wavenumber here taken as identical to $k_{p}$ and $\omega$ in this equation also defined in terms of $k_{p}$ using the standard deepwater linear dispersion equation (see Toffoli et al. [26] for the equation in this form. The left-hand side of this equation is an approximation to the linear dispersion equations. The right-hand side contains non-linear terms. The final term in equation 8 accounts for the interaction between the wave-group and the induced current. The potential $\phi$ satisfies

$$
\left.\frac{\partial \phi}{\partial z}\right|_{z=0}=\frac{\omega}{2} \frac{\partial|A|^{2}}{\partial x}
$$

and within the fluid

$$
\nabla^{2} \phi=0
$$

with $\phi$ going to zero in the limit as $z \rightarrow-\infty$. 
The final term in equation 8 can conveniently be written in terms of the Hilbert transform of the wave envelope [27, 28].

Equation 8 is derived under the assumption that wave steepness is $\mathcal{O}(\epsilon)$ and the bandwidth is $\mathcal{O}\left(\epsilon^{1 / 2}\right)$. The initial conditions for the simulations here $\left(a k_{p}=0.18\right.$ and $s_{x} / k_{p}=$ 0.16 ) would be expected to satisfy these assumptions.

We solve equation 8 using a pseudo-spectral scheme to evaluate the linear part, solving the right-hand side in the spatial domain. We time-march the equation using a 4 th order Runge-Kutta scheme with a timestep of $0.5 \mathrm{~s}$. The spatial discretisation used is $5 \mathrm{~m}$. Filtering is applied to wavenumbers larger than 5 times the spectral peak - due to unphysical transfers of energy to high frequencies (see [29]) this make very minor changes the results for the most non-linear cases presented here. In all cases presented here energy is conserved to within $0.5 \%$. We have also checked that the code can be accurately run forwards through the largest event and then backwards in time to the initial conditions. This is a sensitive chack on the internal consistency of the numerical solution.

In this paper we are going to be interested in splitting the wave into its free wave and bound wave constituents. The distinction is that the free waves move with their own dynamics whereas the bound waves are functions of the free waves. For the envelope equations this splitting is straightforward as these are evaluated separately. For the fully non-linear simulations we use the approximate approach developed by [30] (see also [31]). We use simulations which are $180^{\circ}$ out of phase with each other and use this to split the signal into odd and even harmonics. Consider a perturbed Stokes wave in time with linear components $\eta_{l}=A(t) \cos (\psi)$. Due to the presence of bound harmonics this would produce a free surface

$$
\eta_{0}=A(t) \cos (\psi)+A(t)^{2}\left(\beta^{22} \cos (2 \psi)+\beta^{20}\right)+A(t)^{3}\left(\gamma^{31} \cos (\psi)+\gamma^{33} \cos (3 \psi)\right)+\mathcal{O}\left(A^{4}\right),
$$

where $\beta$ and $\gamma$ are coefficients. If we apply a $180^{\circ}$ phases shift (i.e. simply negating $A(t)$ ) this becomes

$$
\eta_{180}=-A(t) \cos (\psi)+A(t)^{2}\left(\beta^{22} \cos (2 \psi)+\beta^{20}\right)-A(t)^{3}\left(\gamma^{31} \cos (\psi)+\gamma^{33} \cos (3 \psi)\right)+\mathcal{O}\left(A^{4}\right) .
$$

Subtracting $\left(\eta_{0}-\eta_{180}\right)$ (and halving) gives odd harmonics

$$
\eta_{\text {odd }}=A(t) \cos (\psi)+A(t)^{3}\left(\gamma^{31} \cos (\psi)+\gamma^{33} \cos (\psi)\right)+\mathcal{O}\left(A^{5}\right),
$$

whilst adding gives even harmonics in a similar way. For narrow banded spectra, different constituents may then be separated by standard filtering. This technique has been applied to all the fully non-linear simulations in this paper. Therefore the comparisons are between the free waves predicted by the different models. A comparison of bound waves predictions are given in [16] for directionally spread waves - for bound waves we would expect similar agreement in uni-directional waves.

\section{Results}

\subsection{Spatial changes}

Perhaps the most important result is whether the approximate equations reproduce the same maximum amplitudes during the runs. Figure 2 presents the non-linear amplification of 


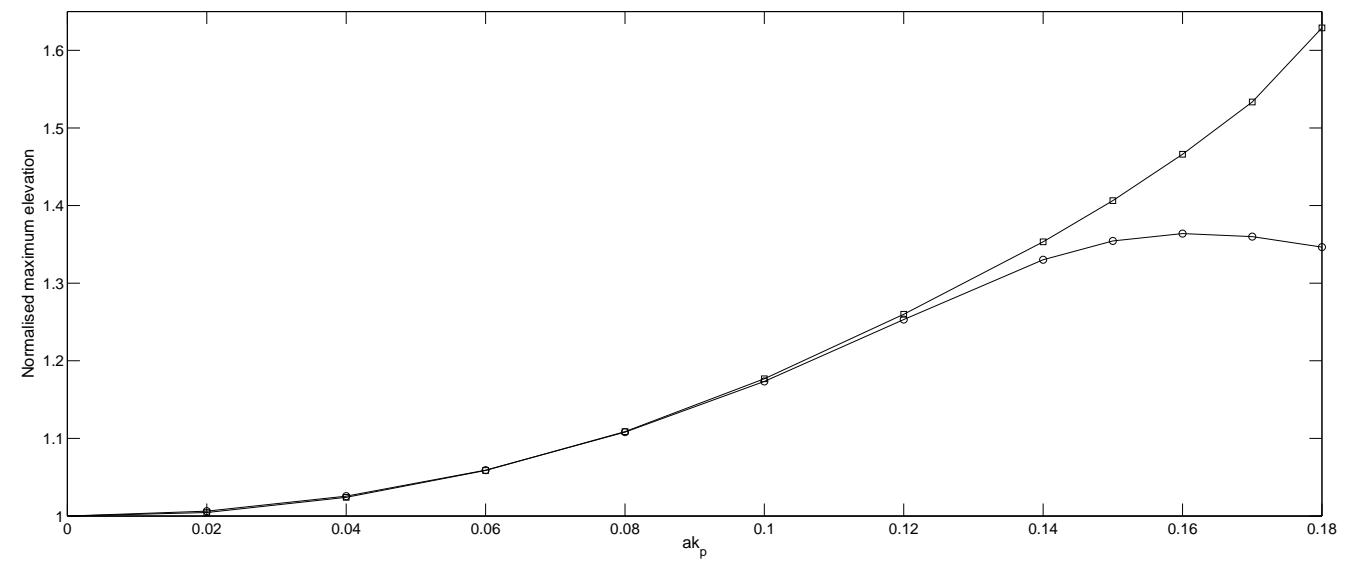

Figure 2: Maximum elevation of linear components in non-linear run normalised by maximum in linear run. Squares - fully non-linear; circles - MNLS.

the wave crest by plotting maximum linearised elevation recorded during the run, normalised by the amplitude that would be reached under linear evolution.

There are two distinct parts to these results. For linear focus amplitude values less than $\sim 0.13 a k_{p}$ the MNLS accurately matches the extra elevation predicted by the fully non-linear results. For amplitudes greater than $\sim 0.13 a k_{p}$ there is a different trend. The MNLS now under-predicts the size of the largest waves and in fact for the MNLS extra input non-linearity does not lead to increasing non-linear amplification of the resulting wave.

From the above it appears that there is a critical point at which the approximations inherent in the MNLS equation breakdown and it stops being a satisfactory model. The steepness of the underlying wave at the point where the MNLS approximation has broken down has increased above that expected under linear evolution, but is only $\sim 0.17$. This implies that it is probably the bandwidth constraint which is the issue. If we examine the non-linear case in Figure 1 the region of the envelope peak has become very sharp - almost forming a cusp in space - which can be thought of as a feature with an extremely broad localised bandwidth. As such it is this local non-linear increase in the spatially localised bandwidth which is causing the adequacy of the results, as a representation of how the potential flow equations behave, to break down.

The increase in the localised bandwidth of wave-groups was reported in the thesis of Gibbs [22] and are reproduced in Figure 3a. Gibbs analysed this by fitting a Gaussian to the peak of the spatial wave-group. The bandwidth can then be found by considering the bandwidth of a Gaussian wave-group with this shape. The Gaussian shape is given by

$$
A_{\text {max }} \exp \left(-\frac{1}{2} s_{x}^{2} x^{2}\right)
$$

The 'local' bandwidth is determined from

$$
s_{x}=\sqrt{\frac{\pi}{2}} \frac{d \hat{A}}{d x} \frac{1}{\sqrt{A^{2}+\hat{A}^{2}}},
$$



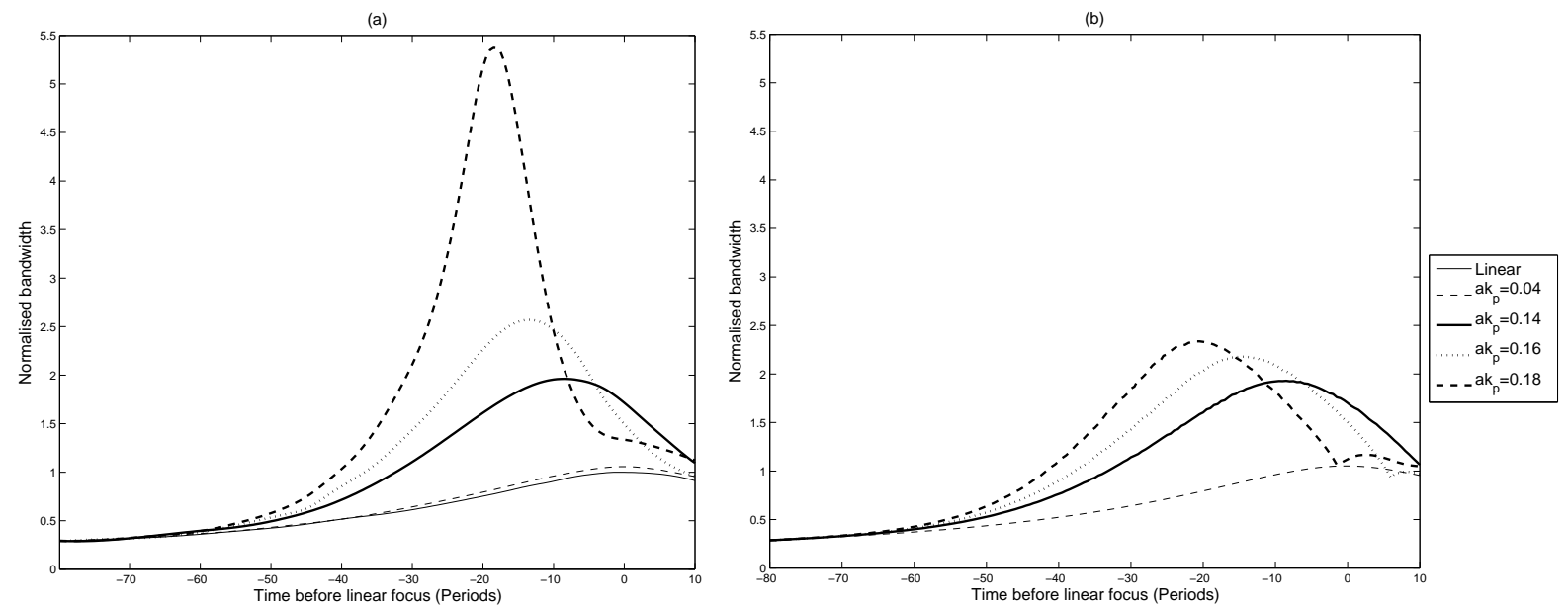

Figure 3: Bandwidth changes as wave-groups focus and de-focus. (a) Fully non-linear; (b) MNLS. Normalised bandwidth defined as local bandwidth divided by local bandwidth at linear focus.

evaluated at the maximum of the 'envelope of the envelope' given by $\sqrt{A^{2}+\hat{A}^{2}}$ where $A$ is the envelope of the linearised free surface and $\hat{A}$ the Hilbert transform of the envelope of the linearised free surface. This formulation is discussed in the Appendix.

Figure 3a shows the dramatic change in the local bandwidth (an increase of more than a factor of 5), and hence the shape of the wave-group, under non-linear evolution. The MNLS also predicts significant non-linear contraction of the wave-group but for the most non-linear case considered this is less than half the change predicted by the fully non-linear simulations (Figure 3b). Thus what is being shown here is that non-linear physics is driving a local increase in the bandwidth and that, for the more non-linear cases, this leads to both the changes and group shape being poorly modelled by the MNLS.

In Figure 4 we examine what happens spatially to the wave-group around the point at which the MNLS starts to break down. The dominant changes to group shape are the same as already presented in Figure 1. The MNLS captures both the contraction in the group width and the movement of the largest wave towards the front of the wave-group. There is only one significant difference between the two cases which is that the oscillations in front of the wave-group are significantly more pronounced in the slightly more non-linear case. The MNLS is predicting an unphysical transfer of energy to these wiggles which are not present in the fully non-linear simulations due to the underlying limitations of the MNLS.

It is interesting that both the MNLS simulation and also in the high order spectral scheme both produce the highest wave elevation and most compact wave packet well before linear focus. Clearly the nonlinear dynamics are interacting with the linear focus process to speed this up during the period when the energy field is becoming more concentrated. Detailed analysis by Gibbs [22] shows that the phase speed of wavenumber components close to the peak of the energy spectrum increases as the group starts to focus, and this effect may drive the focus event to occur earlier. The increase of phase speed with wave steepness 

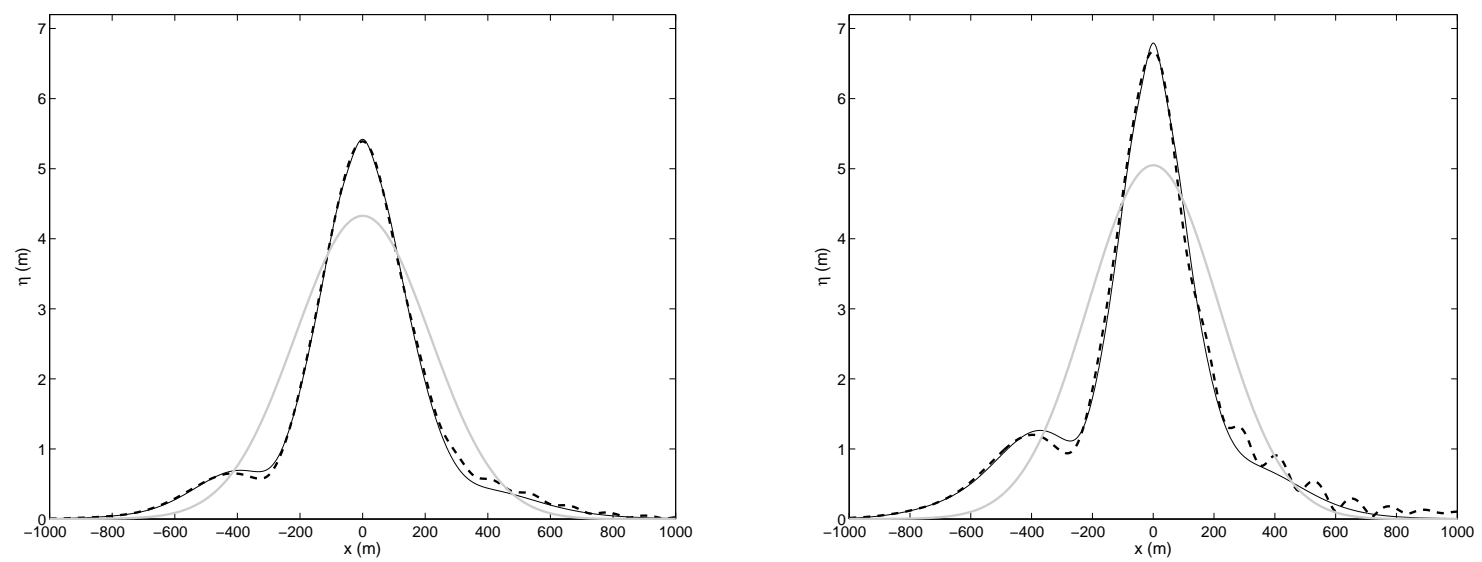

Figure 4: Envelopes of the wave-groups at focus. Left $a k_{p}=0.12$; right $a k_{p}=0.14$. Thick grey - linear; thick black dash - MNLS; thin black line - fully non-linear.

is, of course, a familiar effect for a regular Stokes wave train on deep water.

\subsection{Spectral changes}

As well as considering spatial changes to the wave-group we can examine the changes to the spectrum. Figure 5 shows the changes to the amplitude spectrum at four different points during the evolution for a wave which under linear evolution would have steepness $a k_{p}=0.16$.

Both models predict similar overall behaviour. The initial linear spectrum is modified as the wave-group focuses with a downshift of the spectral peak and a broadening of the spectrum. As the wave-group passes through focus this broadening continues and a clear double peaked structure is formed (we note that this is similar to the spectrum of the famous Draupner New Year Wave if only several wave periods either side of the wave are considered [32]). As the group de-focuses the spectrum resembles its original shape although with some small energy transfers and an increase in the sum of amplitude components (described in greater detail in Gibbs [22]).

Both models predict very similar changes to the spectrum, despite this being for a case $\left(a k_{p}=0.16\right)$ where the peak amplitude in the run is substantially under-predicted by the MNLS. A key feature present in the MNLS solution is the unphysical 'leakage' of energy to wave-numbers at 3 times the (downshifted) spectral peak which is a well known feature of the numerical solutions to the standard NLS-type equations (see Martin \& Yuen's classic paper [29]).

\section{Discussion}

The results presented here show that up until a given non-linearity the MNLS is an excellent model for the evolution of uni-directional waves. Given the limitations in the derivation of the MNLS this is an unsurprising result. However, the point we wish to 

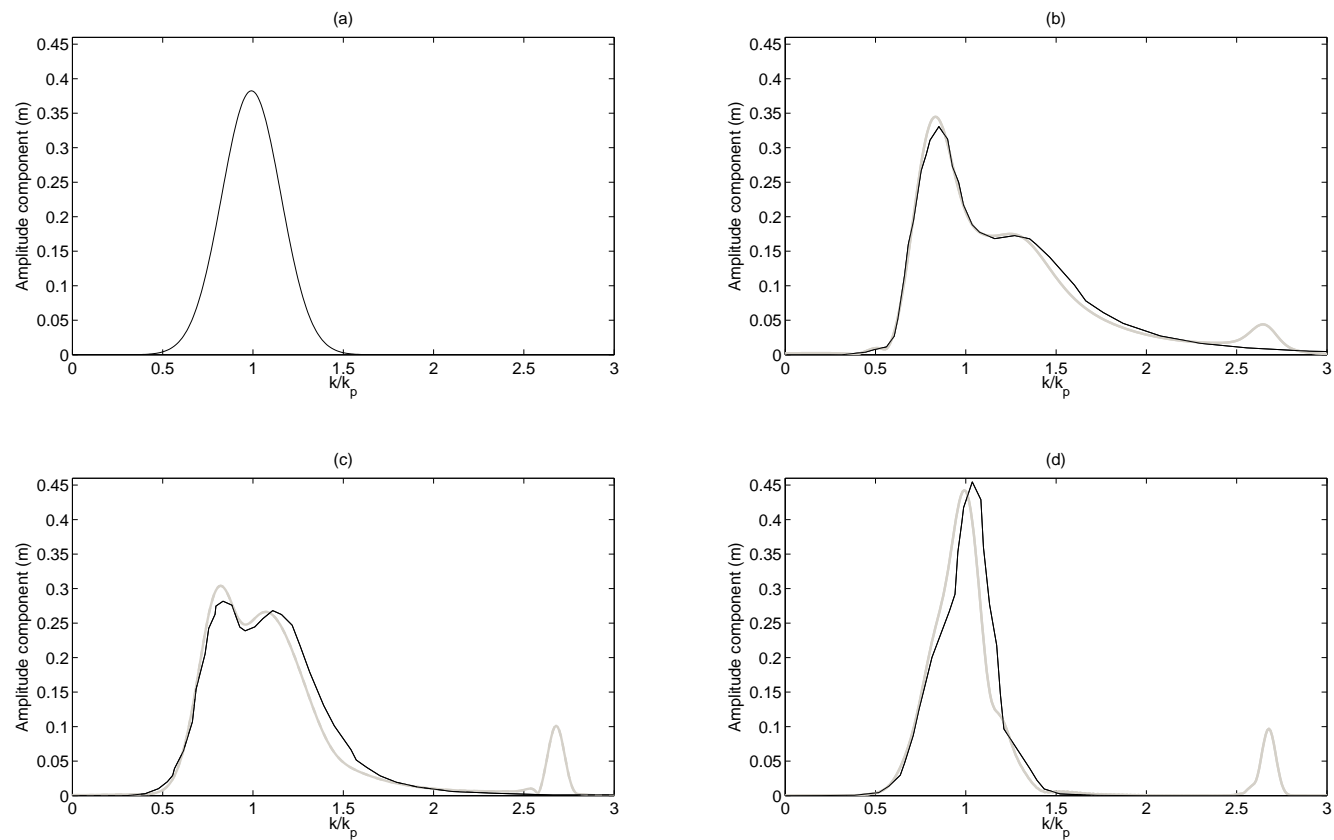

Figure 5: Amplitude spectra. Black thin line - fully non-linear; grey thick line - MNLS. (a) 80 periods before linear focus (linear spectrum - identical for both models); (b) 17.1 periods before linear focus (non-linear focus); (c) 1.1 periods before linear focus; (d) 80.2 periods after linear focus. 
emphasise from this study is that this limitation is not based on the spectrum or degree of non-linearity used to initialise a simulation but on the non-linearity the model needs to simulate during the run. In uni-directional seas non-linear physics drives not only an increase in wave steepness, but also a dramatic increase in localised bandwidths. There will always be an interplay between these two measures of non-linearity but for the case we consider here it is noticeable that the model breaks down for an apparently mild linear steepness of $\sim a k_{p}=0.13$. This suggests there are quite severe limitation on using the MNLS to simulate large waves in even moderately non-linear uni-directional sea-states. By contrast, in sea-states with realistic directional spreads, whilst the MNLS cannot simulate some of the local properties of large waves, the model gives a good representation of the non-linear changes to the shape of a wave-group for waves whose initial conditions are much more non-linear than the limiting non-linearity found in this study [16].

The results presented here are for isolated wave-groups. In many circumstances we might be interested in random waves. Although we have here considered wave-groups based on the average shape of extreme waves in ocean, even under linear evolution extreme waves show a significant variation in shape for the same underlying spectrum [33]. Caution must therefore be used when applying the results herein to random wave simulations but they may be taken as a reasonable guide if non-linearity is estimated from the steepness of the largest wave.

\section{Conclusions}

This paper presents a comparison of two models of non-linear evolution of uni-directional waves on deep water. We have compared potential flow simulations of isolated 'NewWave' type wave-groups with simulations made using the Broad-banded Modified Non-linear Schrödinger equation.

As uni-directional wave-groups focus they undergo substantial non-linear changes. These include an amplification of the amplitude of the wave-group and an increase in the local bandwidth of the wave-group. These changes are quite fast, and would certainly be visible in wave tank experiments. For mild non-linearities, the MNLS accurately matches the results of the potential flow simulations. However, once the non-linear changes to the wave-group lead to the wave-group becoming (locally) broadbanded the MNLS fails to accurately model the detailed spatial structure of the wave-group including substantially underestimating the increase in the amplitude of the wave-crest. Despite this the MNLS still does a reasonable job of simulating spectral changes over the entire focus event, apart from a small amount of leakage of energy to high wavenumbers.

\section{Acknowledgement}

We are grateful to Dr Richard Gibbs for his work on the 'fully non-linear' simulations used in this paper. 


\section{References}

[1] D. Clamond, M. Francius, J. Grue, C. Kharif, Long time interaction of envelope solitons and freak wave formations, European Journal of Mechanics-B/Fluids 25 (5) (2006) 536-553.

[2] K. L. Henderson, D. H. Peregrine, J. W. Dold, Unsteady water wave modulations: fully nonlinear solutions and comparison with the nonlinear Schrödinger equation, Wave motion 29 (4) (1999) 341361.

[3] L. Shemer, B. Dorfman, Experimental and numerical study of spatial and temporal evolution of nonlinear wave groups, Nonlinear Processes in Geophysics 15 (6) (2008) 931-942.

[4] E. Lo, C. C. Mei, A numerical study of water-wave modulation based on a higher-order nonlinear Schrödinger equation, Journal of Fluid Mechanics 150 (1985) 395-416.

[5] L. Shemer, E. Kit, H. Jiao, An experimental and numerical study of the spatial evolution of unidirectional nonlinear water-wave groups, Physics of Fluids 14 (10) (2002) 3380-3390.

[6] T. E. Baldock, C. Swan, P. H. Taylor, A laboratory study of nonlinear surface waves on water, Philosophical Transactions of the Royal Society of London A: Mathematical, Physical and Engineering Sciences 354 (1707) (1996) 649-676.

[7] T. B. Johannessen, C. Swan, A laboratory study of the focusing of transient and directionally spread surface water waves, Proceedings of the Royal Society of London A: Mathematical, Physical and Engineering Sciences 457 (2008) (2001) 971-1006.

[8] R. H. Gibbs, P. H. Taylor, Formation of walls of water in 'fully' nonlinear simulations, Applied Ocean Research 27 (3) (2005) 142-157.

[9] R. S. Gibson, C. Swan, The evolution of large ocean waves: the role of local and rapid spectral changes, Proceedings of the Royal Society of London A: Mathematical, Physical and Engineering Sciences 463 (2077) (2007) 21-48.

[10] S. Yan, Q. W. Ma, QALE-FEM for modelling 3D overturning waves, International Journal for Numerical Methods in Fluids 63 (6) (2010) 743-768.

[11] T. A. A. Adcock, P. H. Taylor, Focusing of unidirectional wave groups on deep water: an approximate nonlinear Schrödinger equation-based model, Proceedings of the Royal Society of London A: Mathematical, Physical and Engineering Sciences 465 (2110) (2009) 3083-3102.

[12] T. A. A. Adcock, R. H. Gibbs, P. H. Taylor, The nonlinear evolution and approximate scaling of directionally spread wave groups on deep water, Proceedings of the Royal Society of London A: Mathematical, Physical and Engineering Sciences 468 (2145) (2012) 2704-2721.

[13] M. J. Lighthill, Contributions to the theory of waves in non-linear dispersive systems, J. Inst. Maths Applics 1 (1965) 269-306.

[14] G. B. Whitham, A general approach to linear and non-linear dispersion using a Lagrangian, J. Fluid Mech. 22 (1965) 273-283.

[15] T. A. A. Adcock, P. H. Taylor, The physics of anomalous ('rogue') ocean waves, Reports on Progress in Physics 77 (10) (2014) 105901.

[16] T. A. A. Adcock, P. H. Taylor, Fast and local non-linear evolution of steep wave-groups on deep water: A comparison of approximate models to fully non-linear simulations, Physics of Fluids 28 (1) (2016) 016601.

[17] G. Lindgren, Some properties of a normal process near a local maximum, The Annals of Mathematical Statistics (1970) 1870-1883.

[18] P. Boccotti, Some new results on statistical properties of wind waves, Applied Ocean Research 5 (3) (1983) 134-140.

[19] T. A. A. Adcock, P. H. Taylor, S. Draper, Non-linear dynamics of wave-groups in random seas: Unexpected walls of water in the open ocean, Proceedings of the Royal Society of London A: Mathematical, Physical and Engineering Sciences 2015 (471).

[20] H. Socquet-Juglard, K. Dysthe, K. Trulsen, H. E. Krogstad, J. Liu, Probability distributions of surface gravity waves during spectral changes, Journal of Fluid Mechanics 542 (2005) 195-216.

[21] W. J. D. Bateman, C. Swan, P. H. Taylor, On the efficient numerical simulation of directionally spread surface water waves, Journal of Computational Physics 174 (1) (2001) 277-305. 
[22] R. H. Gibbs, Walls of Water on the Open Ocean, DPhil Thesis, University of Oxford (Trinty term 2004).

[23] D. G. Dommermuth, D. K. P. Yue, A high-order spectral method for the study of nonlinear gravity waves, Journal of Fluid Mechanics 184 (1987) 267-288.

[24] K. B. Dysthe, Note on a modification to the nonlinear Schrodinger equation for application to deep water waves, Proceedings of the Royal Society of London. A. Mathematical and Physical Sciences 369 (1736) (1979) 105-114.

[25] K. Trulsen, K. B. Dysthe, A modified nonlinear Schrödinger equation for broader bandwidth gravity waves on deep water, Wave motion 24 (3) (1996) 281-289.

[26] A. Toffoli, O. Gramstad, K. Trulsen, J. Monbaliu, E. Bitner-Gregersen, M. Onorato, Evolution of weakly nonlinear random directional waves: laboratory experiments and numerical simulations, Journal of Fluid Mechanics 664 (2010) 313-336.

[27] P. A. E. M. Janssen, On a fourth-order envelope equation for deep-water waves, Journal of Fluid Mechanics 126 (1983) 1-11.

[28] T. S. van den Bremer, P. H. Taylor, Estimates of Lagrangian transport by surface gravity wave groups: The effects of finite depth and directionality, Journal of Geophysical Research: Oceans 120 (4) (2015) $2701-2722$.

[29] D. U. Martin, H. C. Yuen, Quasi-recurring energy leakage in the two-space-dimensional nonlinear Schrödinger equation, Physics of Fluids 23 (5) (1980) 881-883.

[30] P. H. Taylor, D. G. Walker, R. Eatock Taylor, A. C. Hunt, On the estimation of directional spreading from a single wave staff, in: Proc. 5th Int. Conf. on Ocean Wave Measurement and Analysis, Madrid, Spain, 2005, pp. 3-7.

[31] C. J. Fitzgerald, P. H. Taylor, R. Eatock Taylor, J. Grice, J. Zang, Phase manipulation and the harmonic components of ringing forces on a surface-piercing column, Proceedings of the Royal Society of London A: Mathematical, Physical and Engineering Sciences 470 (2168) (2014) 20130847.

[32] T. A. A. Adcock, P. H. Taylor, S. Yan, Q. W. Ma, P. A. E. M. Janssen, Did the Draupner wave occur in a crossing sea?, Proceedings of the Royal Society of London A: Mathematical, Physical and Engineering Sciences 467 (2134) (2011) 3004-3021.

[33] T. A. A. Adcock, S. Draper, A note on the variation in shape of linear rogue waves in the ocean, Underwater Technology 33 (2) (2015) $1-6$.

\section{Appendix}

In this paper we choose to evaluate a localised bandwidth (following Gibbs \& Taylor $[22,8])$ from equation 15 . This is derived as follows:

We define the Gaussian with a bandwidth parameter $s$ as

$$
G=A \exp \left(-\frac{1}{2} s^{2} x^{2}\right),
$$

This has the Hilbert transform

$$
\hat{G}=A \frac{2}{\sqrt{\pi}} F\left(\frac{s x}{\sqrt{2}}\right),
$$

where $F$ is the Dawson function. Differentiating this and evaluating at $x=0$ gives

$$
\left.\frac{d \hat{G}}{d x}\right|_{x=0}=A \sqrt{\frac{2}{\pi}} s .
$$


It is straightforward to evaluate the left hand-side numerical for a given simulation and then solve for the bandwidth.

The locally envelope of the wavegroup in our simulations is not exactly symmetric about a vertical line of symmetry passing through the position of its peak and so its Hilbert transform, $\hat{A}$, will not be exactly zero at this position. Therefore the derivative of $\hat{A}$ will have a maximum that will not exactly coincide with the position of the peak of the envelope, $A$, and so the peak value of the envelope is determined from the 'envelope of the envelope', $\sqrt{A^{2}+\hat{A}^{2}}$.

This approach does not give identical answers to an approach based on double differentiation (except for a perfect Gaussian) and we therefore urge caution in using it. We use the approach above here as it only requires a single differentiation and is therefore much less susceptible to noise than an approach based on differentiating twice. In the estimation of the local linearised wave envelope contained within the fully nonlinear solution, there is some residual spillover across high wavenumbers which cannot be removed by simple filtering, and this renders double differentiation problematical. 